\title{
AVALIAÇäO DA COMPOSIÇÃO CORPÓREA E DENSIDADE MINERAL ÓSSEA EM MULHERES PORTADORAS DE ARTRITE REUMATÓIDE
}

Raíssa Gomes da Silva*, Maria Guadalupe Barbosa Pippa, Cristiano Augusto de freitas Zerbin

Trabalho realizado pela Disciplina de Reumatologia da Faculdade de Medicina da Universidade de São Paulo e Serviço de Reumatologia do Hospital Heliópolis, São Paulo/SP.

\author{
*Correspondência: \\ Av. Rebouças, 1101 \\ São Paulo/SP \\ Cep: 05401-150 \\ raissajp@gmail.com
}

\begin{abstract}
RESUMO
OBjetivo. Analisar o comprometimento da densidade mineral óssea (DMO) e a sua correlação com a composição corporal em mulheres com AR.

Métodos. Oitenta e três pacientes com AR realizaram densitometria óssea (DXA) para análise de massa óssea total e regional, e estudo da composição corpórea (CC). Além disso, foram submetidas a radiografia lateral de coluna dorsal e lombar e exames laboratoriais. Foram aplicados questionários para avaliação da atividade da doença, classe funcional, atividade física e inquérito alimentar.

Resultados. A prevalência de osteoporose nas pacientes menopausadas foi de 21,4\% (I2 pacientes), 46,4\% com osteopenia (26 pacientes) e 32, $1 \%$ com valores normais ( 18 pacientes) e a osteoporose ocorreu de forma semelhante em coluna lombar e colo do fêmur. Mais da metade da amostra apresentou diminuição de DMO em coluna lombar e lou colo do fêmur. As mulheres de raça não branca e as pré-menopausadas apresentaram maiores valores nas médias de DMO. A dose cumulativa de GC nos últimos dois anos foi determinante negativo na massa magra total. A idade teve efeito negativo nas medidas DMO e de CC. O IMC mostrou efeito positivo em todas as variáveis da CC. As classes funcionais 3 e 4 tiveram efeito negativo apenas na DMOT. A atividade física apresentou efeito positivo na DMOFT. A duração da AR teve efeito negativo na DMOCL. A dose de GC dos últimos três meses mostrou efeito negativo na MMT e a dose dos últimos dois anos teve efeito positivo no PGT. Por fim, o TEE teve efeito positivo no PGT.

Conclusão. A redução da DMO observada em $67,8 \%$ das pacientes sugere a melhoria de medidas para a prevenção e tratamento de osteoporose a partir do diagnóstico desta doença. A idade e raça branca foram fatores negativos nas medidas de DMO, enquanto que o IMC foi um fator positivo em todas as variáveis de DMO e CC. A doença (AR) também influenciou negativamente a DMO nestas pacientes, e a utilização de GC modificou a CC, reduzindo a massa muscular e aumentando o percentual de gordura nestas pacientes. Embora não tenhamos evidenciado uma ação deletéria direta do GC na DMO, o seu uso deve ser criterioso. A preservação da massa muscular e o incentivo de seu desenvolvimento são importantes no equilibrio das pacientes, com conseqüente diminuição de quedas e futuras fraturas.
\end{abstract}

UnITERMOS: Composição corpórea. Densidade mineral óssea. Artrite reumatóide. Osteoporose.

\section{INTRODUÇão}

As aferições da composição corpórea (CC) são de grande importância para o diagnóstico e controle de várias enfermidades. A absorciometria por raios $X$ de dupla energia (DXA), técnica mais utilizada para avaliação da densidade mineral óssea (DMO), tem sido aplicada com sucesso na mensuração da composição corpórea, visto que separa o osso da massa gorda e massa magra!.

Assim como o envelhecimento, as doenças agudas e crônicas promovem diminuição da massa magra. Este processo sofre influência da alteração na produção hormonal, de citocinas e da atividade física².

A artrite reumatóide (AR) é uma doença inflamatória crônica, marcada por extenso comprometimento da membrana sinovial. Quando inflamada, a sinovial libera citocinas que causam prejuízo cartilaginoso, justa-articular e ósseo. Novas evidências sugerem que na AR a destruição óssea local e a sistêmica são mediadas por uma via comum: a ativação de osteoclastos ${ }^{3}$.

A maioria dos estudos que avalia a DMO na AR indica que ocorre também uma perda generalizada, sendo mais evidente em quadril e rádio do que na coluna. A sua patogênese é multifatorial e medicações habitualmente utilizadas na AR também estão envolvidas no mecanismo de perda óssea nestes pacientes'. A perda óssea na AR é marcadamente pronunciada nos pacientes tratados com glicocorticóides (GC), sendo que a osteoporose induzida pelo uso de GC é a principal causa secundária desta enfermidade em homens e mulheres. $O$ efeito do GC na massa óssea já ocorre nos primeiros meses de tratamento. $\mathrm{O}$ aumento do risco de fratura associado ao uso deste fármaco é bem definido. Estima-se que mais de $50 \%$ dos pacientes cronicamente tratados com GC irão sofrer fraturas ao longo da vida, e a incidência de fraturas é de aproximadamente 30\% após cinco anos de tratamento ${ }^{2,3}$. 
SILVA RG ET AL.

O GC afeta primariamente a formação óssea suprimindo o número, função e meia-vida dos osteoblastos, mas também tem efeito na reabsorção óssea devido ao aumento da atividade dos osteoclastos ${ }^{4}$. O aumento da reabsorção óssea nos usuários de GC também pode ser explicado em parte pelo hiperparatireoidismo secundário e pelo seu efeito inibitório na glândula pituitária e gônadas ${ }^{5}$.

A literatura dispõe de poucos estudos que analisam a composição corporal na AR. Uma reduzida massa magra foi detectada inicialmente por dados antropométricos e impedância bioelétrica por Roubenoff e cols. em uma pequena amostra de 24 pacientes com AR. Os autores observaram que a caquexia é comum na AR (presente em 67\% destes), e que a massa magra corporal foi inversa e significativamente associada ao número de articulações edemaciadas ${ }^{4}$.

Westhovens et al. estudaram 98 pacientes com artrite reumatóide e confirmaram uma significante redução da massa magra em todos os sítios analisados quando comparados aos controles. De forma inversa, os autores encontraram um aumento da massa gorda, com distribuição central, sugerindo que este achado poderia ser um preditor para doença cardiovascular².

Posteriormente, em 1996, Hernadez-Beriain et al., utilizando dados antropométricos, avaliaram o estado nutricional de 75 pacientes portadores de AR, com um tempo de doença médio de I0 anos e demonstraram que houve uma significante perda de massa magra nos pacientes com AR, particularmente naqueles com maior incapacidade funcional (classes III e IV) .

Utilizando densitometria, Sambrook et al. avaliaram 12 pares de gêmeos monozigóticos, onde um dos pares era portador de AR. A MMT e a massa magra das pernas estavam significativamente reduzidas (média de 5,2\% a 12,3\%) no gêmeo com AR. No entanto, não houve diferença na MGT, peso ou altura entre os pares. Os autores acreditam que esses achados sejam resultado da atividade física reduzida com conseqüente diminuição da atividade muscular nos indivíduos doentes 6 .

São poucos os estudos que avaliam a relação entre DMO e composição corporal em pacientes com AR. Visto que a AR é uma enfermidade com prevalência significativa, e associado ao fato destes pacientes apresentarem importante prejuízo da massa óssea, realizamos este estudo.

\section{Métodos}

Este é um estudo transversal. Analisamos 120 mulheres com diagnóstico de AR segundo os critérios classificatórios do American College of Rheumatology (ACR) ${ }^{7}$. Os critérios de exclusão foram a presença de qualquer hábito ou doença que sabidamente interferisse no metabolismo ósseo. Destas, 37 foram excluídas, sendo que 30 por não preencherem os critérios necessários para esta pesquisa e as outras sete por dificuldade de locomoção.

Questionários: As pacientes incluídas no estudo responderam os questionários listados abaixo.

- European Vertebral Osteoporosis Study (EVOS) tem como objetivo determinar a prevalência e fatores de riscos relacionados à deformidade vertebral ${ }^{8}$.
- Para a avaliação da atividade física, utilizou-se o questionário de atividade física habitual de Baecke?.

- Questionário de inquérito alimentar aplicado por uma nutricionista já validado ${ }^{10}$.

Na avaliação clínica, utilizamos o cálculo do Disease Assessment Score (DAS28)" e Health Assessment Questionnare $(\mathrm{HAQ})^{12}$. Obtivemos o tempo de duração da doença, classe funcional segundo os critérios de Steinbrocker ${ }^{13}$ e registramos as medicações em uso. O cálculo da dose de GC foi obtido em relação à sua equivalência à prednisona. A dose atual foi calculada como a média em mg utilizada nos últimos três meses. A dose cumulativa correspondeu à soma em mg de todo GC utilizado, incluindo aqueles usados em infiltração articular durante os últimos dois anos.

Durante a avaliação laboratorial, procedemos a análise de proteína $C$ reativa (PCR), velocidade de hemossedimentação (VHS) e dosagem de fator reumatóide (FR).

Em uma segunda consulta, realizamos a avaliação da composição corporal e da DMO de corpo total, coluna e femoral, por meio de um densitômetro de dupla emissão de raios $X$, modelo DPX IQ (Lunar Corp., Madison,WI, fabricado nos Estados Unidos), assim como radiografia lateral de coluna torácica e lombar para classificação de fraturas de acordo com os critérios semiquantitativos de Genant ${ }^{14}$. Foram estudadas a densidade mineral óssea total (DMOT), densidade mineral óssea de colo femural (DMOCF), densidade mineral óssea de fêmur total (DMOFT) e densidade mineral óssea de coluna lombar (DMOCL), incluindo as medidas de CC, ou melhor, massa magra total (MMT), massa gorda total (MGT) e percentual de gordura total (PGT). Foram utilizados os critérios da Organização Mundial da Saúde para a classificação de osteoporose, osteopenia e normal para as pacientes menopausadas ${ }^{15}$. O coeficiente de variação das medidas densitométricas na Unidade de Densitometria do PAM Heliópolis foi inferior a 1,5\% em todas as áreas estudadas. Segundo os critérios semiquantitativos de Genant, classifica-se como grau 0 ou normal a ausência de deformidade vertebral, grau I a redução de $20 \%$ a $25 \%$ na altura vertebral (anterior, média ou posterior), grau 2 a redução de $25 \%$ a $40 \%$ na altura vertebral (anterior, média ou posterior) e grau 3 a redução maior de $40 \%$ na altura vertebral (anterior, média ou posterior). Foi classificada a raça como branca (BR) pela ausência da raça negra nos familiares de primeira geração, e raça não branca (NBR) de acordo com auto-denominação da paciente.

A caracterização da amostra foi feita por meio de proporções, média, desvio padrão, mediana e valores mínimos e máximos. Todas as variáveis estavam distribuídas normalmente. Para comparar as diferenças entre variáveis da CC, foram utilizados o teste de t de Student ou análise de variância a um fator (Anova). A correlação entre as variáveis foi estimada usando o coeficiente de correlação de Pearson ( $r$ ). Para fazer a análise conjunta dos fatores associados à CC das pacientes $A R$, foi utilizada a análise de regressão linear múltipla. Foi considerada variável dependente cada uma das medidas da CC e DMO e conteúdo mineral ósseo. 


\section{Resultados}

Do total de 83 mulheres que participaram do estudo, 57 $(68,7 \%)$ eram brancas (BR) e 26 (31,3\%) não brancas (NBR). A idade média foi de 53,9 anos e o IMC foi $26,8 \mathrm{~kg} / \mathrm{m}^{2}$ (Tabela I). A média de duração da AR foi de 7,73 anos. Quarenta pacientes $(48,2 \%)$ tinham classe funcional $1 ; 15$ classe funcional 2 (I8,1\%); $27(32,5 \%)$ classe funcional 3; e I (1,2\%) classe funcional 4. A média do DAS 28 foi 5,06 e do HAQ 0,960. O FR esteve presente em 59 pacientes $(71,1 \%)$ e o PCR foi positivo em 50 mulheres $(60,2 \%)$. Fratura vertebral foi observada em cinco mulheres (6\%) e todas tinham grau I de Genant. A prevalência de osteoporose entre as pacientes menopausadas (56 pacientes) foi de 21,4\% (12 pacientes) e foi semelhante em coluna lombar e colo do fêmur, 26 $(46,4 \%)$ apresentaram osteopenia e 18 (32,1\%) apresentaram valores normais.

O valor médio das DMO e CC e CMO são mostrados na Tabela I. As mulheres de raça NBR e as pré-menopausa tinham maiores valores das médias de DMOT: $1,09 \mathrm{~g} / \mathrm{cm}^{2} ; p=0,005 \mathrm{e}$ I,094g/ $/ \mathrm{cm}^{2} \quad \mathrm{p}<0,001$, respectivamente.

Para a DMOT, verificamos que foram fatores determinantes independentes a idade $(\beta=-0,0056 ; \quad p<0,001), \quad 0 \quad$ IMC $(\beta=+0,0085 ; p<0,00 \mathrm{I})$, a raça branca $(\beta=-0,0770 ; p<0,00 \mathrm{I})$ e as classes funcionais 3 e $4(\beta=-0,0463 ; p=0,018)$ na análise múltipla. Para cada ano de idade, diminuiu, em média, 0,0056 na DMOT. Para cada I de IMC, houve aumento médio de 0,0085 na DMOT. A raça branca tem, em média, $-0,0770$ de DMOT em relação à raça não branca. As classes funcionais 3 e 4 têm, em média, $-0,0463$ na DMOT em relação à classe funcional.

Houve correlação negativa estatisticamente significativa entre DMOCF e idade $(r=-0,45 ; p<0,001)$ e duração da doença $(r=-$ $0,23 ; \quad p=0,032)$. Esta correlação foi positiva para o IMC $(r=+0,44 ; \quad p<0,00 I)$, dose de corticóide em três meses $(r=+0,25 ; p=0,020)$ e atividade física $(r=+0,25 ; p=0,023)$.
$\mathrm{Na}$ análise múltipla, verifica-se que foram fatores determinantes independentes e significativos para a DMOCF a idade $(\beta=-0,0085$; $p<0,00 \mid)$, o IMC $(\beta=+0,0|34 ; p<0,00|)$ e a raça $B R(\beta=$ $0,0864 ; p=0,005)$. Para cada ano, diminuiu, em média, 0,0085 na DMOCF. Para cada I de IMC, houve aumento médio de 0,0134 na DMOCF. A raça BR tem, em média, -0,0864 de DMOCF em relação à NBR.

A média das DMOFT foi maior nas mulheres de raça NBR $\left(0,943 \mathrm{~g} / \mathrm{cm}^{2} ; p=0,025\right)$. As mulheres que não estavam na menopausa também apresentaram maiores valores de DMOFT $\left(0,934 \mathrm{~g} / \mathrm{cm}^{2} ; p=0,047\right)$. Houve correlação negativa estatisticamente significativa entre DMOFT e idade $(r=-0,40 ; p<0,00$ I) e duração da doença $(r=-0,25 ; p=0,024)$. A atividade física e o IMC apresentaram correlação positiva com a DMOFT: $r=+0,49$; $p<0,001$ e $r=+0,31 ; p=0,004$, respectivamente. Na análise múltipla, verificamos que foram fatores determinantes independentes e significativos para a DMOFT a idade $(\beta=-0,0066 ; p<0,00$ I), - IMC $(\beta=+0,0 \mid 47 ; \quad p<0,00$ I), raça $B R \quad(\beta=-0,077$ I; $p=0,0$ II) e atividade física $(\beta=+0,0269 ; p=0,032)$.

A média de DMOCL mostrou maiores valores nas mulheres $\operatorname{NBR}\left(1,146 g / \mathrm{cm}^{2} \mathrm{p}=0,006\right)$ e naquelas que não estavam na pósmenopausa $\left(1,155 \mathrm{~g} / \mathrm{cm}^{2}, \quad p=0,002\right)$. Também observamos diferença estatisticamente significante na $\mathrm{DMOCL}$ no que se refere à classe funcional $(\mid<2, p=0,008$, e $3<2, p=0,026)$. Houve correlação negativa estatisticamente significativa entre DMOCL e idade $(r=-0,30 ; \quad p=0,006)$ e duração da doença $(r=-0,27$; $p=0,0 \mid 4) . O I M C$ e a atividade física mostraram uma correlação positiva: $r=+0,36 ; p=0,00$ | e $r=+0,27 ; p=0,0 \mid 4$, respectivamente. $\mathrm{Na}$ análise múltipla, verificamos que foram fatores determinantes independentes e significativos para a DMOCL a idade $(\beta=-0,0063 ; p=0,00$ I), o IMC $(\beta=+0,0103 ; p=0,003)$, raça $B R(\beta=-0,1250 ; p=0,002)$ e duração da $A R(\beta=-0,0057$; $p=0,043)$.

\begin{tabular}{|c|c|c|c|c|}
\hline Variável & Média (DP) & Mediana & Mínimo & Máximo \\
\hline Idade(anos) & $53,9(9,86)$ & 55,1 & 27 & 74 \\
\hline Idade de menarca (anos) & $13,2(1,9)$ & 13,0 & 9 & 19 \\
\hline Idade da menopausa (anos) & $46, I(5,2)$ & 45,0 & 31 & 56 \\
\hline Peso $(k g)$ & $64,3(13,4)$ & 63,0 & 37 & 108 \\
\hline Altura $(\mathrm{cm})$ & $154,9(5,6)$ & 155,0 & 139,0 & 170,0 \\
\hline $\operatorname{IMC}\left(\mathrm{kg} / \mathrm{m}^{2}\right)$ & $26,8(19,8)$ & 26,5 & 16,0 & 44,0 \\
\hline Massa gorda (g)total & $20.586, \mid 4(8.637)$ & $1903 \mid$ & 3.432 & 45.538 \\
\hline Massa magra (g)total & $41.227,66(5.482)$ & 41.278 & 26.246 & 55.899 \\
\hline$\%$ gordura total & $32,0(7.81)$ & 31,4 & 8,5 & 44,9 \\
\hline DMO coluna (L2-L4) & $1,062(0,19 \mid)$ & 1,035 & 0,686 & 1,890 \\
\hline DMO colo fêmur $\left(\mathrm{g} / \mathrm{cm}^{2}\right)$ & $0,854(0,169)$ & 0,862 & 0,468 & 1,278 \\
\hline DMO fêmur total $\left(\mathrm{g} / \mathrm{cm}^{2}\right)$ & $0,882(0,168)$ & 0,880 & 0,539 & $|, 3| \mid$ \\
\hline DMO corpo total (DMOT) $\left(\mathrm{g} / \mathrm{cm}^{2}\right)$ & $1,041(0,109)$ & 1,057 & $0,77 \mid$ & 1,320 \\
\hline Conteúdo mineral ósseo $\left(\mathrm{g} / \mathrm{cm}^{2}\right)$ & $2180,51(412,04)$ & 2192 & 1292 & 3488 \\
\hline
\end{tabular}


SILVA RG ET AL.

Ao se analisar a MMT, evidenciamos correlação positiva estatisticamente significativa entre a MMT e IMC $(r=+0,73$; $p<0,00$ I) e ingestão de cálcio $(r=+0,26 ; p=0,0$ |8). Na análise múltipla, verifica-se que foram fatores determinantes independentes e significativos para a MMT a idade $(\beta=-|0|, 503 ; p=0,0 \mid 2)$, - IMC $(\beta=+759,847 ; p<0,00$ I) e dose de GC nos últimos três meses $(\beta=-0,473 ; p=0,008)$. Cada ano de idade diminuiu, em média, I0I,503 na MMT. Para cada I de IMC, houve aumento médio de 759,847 na MMT. Para cada I mg de GC nos últimos três meses, houve diminuição média de 0,473 na MMT.

$\mathrm{Na}$ análise da MGT, observou-se diferença estatisticamente significativa nas médias em relação à classe funcional $(\mid<2$, $p=0,038)$ e ao $F R(F R+>F R ; p=0,0 \mid 3)$. Houve correlação positiva estatisticamente significativa entre MGT e IMC $(r=+0,93$; $p<0,00 I), \quad H A Q \quad(r=+0,23 ; p=0,036)$, DAS $28 \quad(r=+0,22$; $p=0,045)$, dose de $G C$ em três meses $(r=+0,21 ; p=0,052)$ e ingestão de cálcio $(r=+0,29 ; \quad p=0,008)$. $\mathrm{Na}$ análise múltipla, verifica-se que o único fator determinante independente e significativo para a MGT foi o IMC $(\beta=+|478,860 ; p<0,00|)$, sendo que para cada I de IMC, houve aumento médio de I478,860 na MGT. As variáveis idade, dose de GC nos últimos três meses e DAS 28 permaneceram no modelo múltiplo como variáveis de ajuste.

$\mathrm{Na}$ análise do PGT, observamos relação positiva estatisticamente significativa com a dose de GC em três meses $(r=+0,22$; $p=0,044)$, dose de GC acumulada $(r=+0,28 ; p=0,0 \mid I)$, IMC $(r=+0,82 ; \quad p<0,001)$ e ingestão de cálcio $(r=+0,21$; $\mathrm{p}=0,052)$. Na análise múltipla, verificou-se que foram fatores determinantes para o PGT, o IMC $(\beta=+1,|62 ; p<0,00|)$, a dose de $G C$ nos últimos dois anos $(\beta=+0,0008 ; p<0,001)$ e o tempo de exposição ao estrógeno $(\mathrm{TEE})(\beta=+0,164 ; p=0,049)$. As variáveis idade e DAS 28 ficaram no modelo múltiplo final como variáveis de ajuste.
$\mathrm{Na}$ análise múltipla do $\mathrm{CMO}$, verificamos que foram fatores determinantes independentes e significativos para o CMOT a idade $(\beta=+0,|0| ; p=0,033), \quad$ oIMC $(\beta=+1, \mid 76 ; p<0,001)$, a dose de $G C$ nos últimos dois anos $(\beta=+0,0008 ; p<0,00 I)$. A variável DAS ficou no modelo múltiplo como variável de controle.

Resumindo as análises da relação entre DMO, CMO, CC e as características demográficas e clínicas de pacientes com AR observamos que a idade teve efeito negativo nas medidas DMO e de CC. No CMOT, este efeito foi positivo. A raça BR mostrou redução nas medidas de DMO. O IMC mostrou efeito positivo em todas as variáveis da CC. As classes funcionais 3 e 4 tiveram efeito negativo apenas na DMOT. A atividade física apresentou efeito positivo na DMOFT. A duração da AR teve efeito negativo na DMOCL. A dose de GC dos últimos três meses mostrou efeito negativo na MMT e a dose dos últimos dois anos teve efeito positivo no PGT e no CMOT. Por fim, o TEE teve efeito positivo no PGT (Tabela 2).

\section{Discussão}

Este é o primeiro estudo que avaliou a CC em mulheres brasileiras com AR. A exemplo do que ocorre universalmente ${ }^{16,17}$, a DMO desta amostra mostrou correlação inversa em função da idade. A raça BR apresentou correlação negativa com a DMO. $O$ efeito da raça nas medidas de DMO é bastante conhecido, com comprovada influência positiva da raça negra no pico de massa óssea ${ }^{18,19}$. Lembrando que o povo brasileiro é formado por uma variedade de raças que descendem de africanos, europeus e índios, o que resultou em uma população miscigenada, os nossos resultados são semelhantes aos já descritos na literatura ${ }^{18,19}$

Segundo os critérios da Organização Mundial da Saúde ${ }^{20}$, classificamos a média da nossa população como sobrepeso (IMC médio de 26,8). Na análise múltipla, o IMC foi um importante

\begin{tabular}{|c|c|c|c|c|c|c|c|c|}
\hline Variável & $\begin{array}{c}\text { DMOT } \\
\beta(p)\end{array}$ & $\begin{array}{c}\text { DMO colo } \\
\beta(p)\end{array}$ & $\begin{array}{c}\text { DMO quadril } \\
\beta(p)\end{array}$ & $\begin{array}{l}\text { DMO coluna } \\
\beta(p)\end{array}$ & $\begin{array}{l}\text { MMT } \\
\beta(p)\end{array}$ & $\begin{array}{l}\text { MGT } \\
\beta(p)\end{array}$ & $\begin{array}{c}\% \text { gordura } \\
\beta(p)\end{array}$ & СMOT \\
\hline $\begin{array}{l}\text { Idade } \\
\text { Raça branca }\end{array}$ & $\begin{array}{l}-0,0056 \\
(<0,00 \mid) \\
-0,0770 \\
(<0,001)\end{array}$ & $\begin{array}{c}-0,0085 \\
(<0,00 \text { I) } \\
-0,0864 \\
(<0,00 \text { I) }\end{array}$ & $\begin{array}{c}-0,0066 \\
(<0,001) \\
-0,077 \mid \\
(0,011)\end{array}$ & $\begin{array}{l}-0,0063 \\
(0,00 I) \\
-0,1250 \\
(0,002)\end{array}$ & $\begin{array}{c}-101,503 \\
(0,012)\end{array}$ & & & $\uparrow$ \\
\hline $\begin{array}{l}\text { MC } \\
\text { CF } 3+4\end{array}$ & $\begin{array}{c}+0,0085 \\
(<0,00 \mid) \\
-0,0463 \\
(0,0 \mid 8)\end{array}$ & $\begin{array}{l}+0,0134 \\
(<0,001)\end{array}$ & $\begin{array}{l}+0,0147 \\
(<0,001)\end{array}$ & $\begin{array}{c}+0,0103 \\
(0,003)\end{array}$ & $\begin{array}{l}+759,847 \\
(<0,00 I)\end{array}$ & $\begin{array}{c}+\mid 478,860 \\
(<0,00 \mid)\end{array}$ & $\begin{array}{l}+1,162 \\
(<0,001)\end{array}$ & $\uparrow$ \\
\hline Atividade física & & & $\begin{array}{c}+0,0269 \\
(0,032)\end{array}$ & & & & & \\
\hline Duração daAR & & & & $\begin{array}{l}-0,0057 \\
(0,043)\end{array}$ & & & & \\
\hline Dose GC 3 meses & & & & & $\begin{array}{l}-0,473 \\
(0,008)\end{array}$ & & & \\
\hline Dose GC 2 anos & & & & & & & $\begin{array}{l}+0,0008 \\
(<0,00 \mid)\end{array}$ & $\uparrow$ \\
\hline Exposição estrógeno & & & & & & & $\begin{array}{l}+0,164 \\
(0,049)\end{array}$ & \\
\hline
\end{tabular}


preditor da DMO em todos os sítios estudados, sendo estes resultados consistentes com a literatura ${ }^{21}$.

As nossas pacientes apresentaram um tempo médio de doença de 7,73 anos. A perda de massa óssea em coluna lombar e fêmur proximal é descrita em estudos longitudinais, porém a sua relação com o tempo de doença é, às vezes, controversa ${ }^{22,23}$. Nos pacientes cujo tempo de doença é maior que 6 a 12 meses, a perda óssea ocorre de forma mais lenta ${ }^{22}$. Hansen et al. não demonstraram correlação direta entre a DMO axial e apendicular de 95 pacientes com AR e o tempo de doença comparado ao controle ${ }^{23}$.

A funcionalidade avaliada segundo as classes funcionais de Steinbrocker ${ }^{7}$ constitui um instrumento importante, visto que pode refletir o grau de comprometimento decorrente da atividade de doença. Nossos resultados mostraram que as classes funcionais 3 e 4 apresentaram menor DMOT quando comparadas à classe funcional I, o que se assemelha à literatura. Sinigaglia et al. ${ }^{24}$ estudaram 925 mulheres com AR e observaram que a freqüência de baixa DMO aumentou linearmente de acordo com os critérios de classe funcional de I a 4 de Steinbrocker.

Sabe-se que existe uma associação bem estabelecida entre a atividade física habitual, preservação da massa óssea e prevenção de osteoporose ${ }^{25}$. Neste estudo, utilizamos o questionário de Baecke $^{12}$ e nossos resultados mostram uma correlação positiva entre a atividade física e as DMOCL, DMOCF e DMOFT.

Das pacientes, 67,5\% encontravam-se na menopausa. Diminuição da DMO ocorreu em todos os sítios analisados nas mulheres pós-menopausa, conforme o já o visto em literatura ${ }^{26}$. Hall et al. analisaram os marcadores do metabolismo ósseo em 106 mulheres com AR na pós-menopausa, sendo 35 pacientes usuárias de baixa dose de GC e confirmam a relação do status pósmenopausa e a alta remodelação óssea na $A R^{27}$.

A comparação dos valores médios de DMOCL e DMOCF do nosso estudo com uma população brasileira sadia mostrou que as mulheres com AR apresentaram valores 0,56\% e 0,46\% inferiores de DMOCL e DMOCF respectivamente, o que não configura diferença estatística significante ${ }^{28}$.

A maioria dos estudos longitudinais aponta para um maior índice de perda óssea nas pacientes com AR inicial29. Há relato de perda de massa óssea em pacientes com AR quando comparados ao controle, independente do tempo de doença $a^{30}$. Acredita-se que a prevalência de baixa massa óssea na AR seja mais importante do que uma simples comparação de médias de DMO entre os pacientes e o grupo controle. Haugeberg et al. comprovaram esta teoria analisando uma população de 394 pacientes com AR e duração média de doença de 13 anos ${ }^{28}$. Seus resultados mostraram que a prevalência de osteoporose (T score d" -2,5) foi de I4,7\% no colo femural, I4,7\% no fêmur total e 16,85 na coluna. Na análise da redução de massa óssea utilizando o $Z$ score $<-1,0$, estes valores foram maiores do que o esperado: $15,9 \%$ no colo femural, $27,6 \%$ no fêmur total e 19,6\% na coluna.

A relação entre $\mathrm{GC}$ e baixa DMO não é descrita de maneira uniforme entre os relatos ${ }^{29,30}$. De forma não convencional, nosso estudo apresentou correlação positiva entre a dose de GC nos últimos três meses e as DMOT e DMOCF. Resultados semelhan- tes foram descritos por outros autores ${ }^{30}$. É possível que um melhor controle da AR com a terapia com GC tenha melhorado a massa óssea nestes pacientes ou o tamanho da amostra tenha influenciado este resultado.

O CMO é uma importante expressão quantitativa do mineral ósseo e é definido como o conteúdo mineral dentro de uma área, sendo influenciado pelo tamanho do corpo. Sabe-se que o CMO reflete indiretamente a quantidade total de cálcio corpóreo do corpo. Nossos resultados revelam que a dose cumulativa do GC apresentou correlação negativa com o CMO, o que é concordante com a litearatura ${ }^{31}$.

A literatura mostra um aumento da prevalência de baixa massa corporal em pacientes com AR, particularmente a massa magra ${ }^{2,31}$. Obtivemos menores valores médios de MMT quando comparamos com uma população do estudo de Toussirot et al ${ }^{31}$. A relação inversa entre idade e MMT é bem conhecida, sendo que se observa em uma população sadia que a massa magra diminui com a idade ${ }^{32}$.

A correlação do IMC com a massa magra é de fácil compreensão. Sabendo-se que o peso compreende componentes de CC (massa magra e massa gorda) e faz parte do cálculo do IMC, subentende-se que variações neste índice podem ser reflexo de variações da massa magra. $O$ nosso estudo mostrou que para cada I mg de GC usado nos últimos três meses houve diminuição media de 0,473 na MMT. Em recente estudo, Natsui et al. relataram que a massa magra, a qual representa principalmente o volume muscular, diminui rapidamente após o início da terapêutica com GC $\mathrm{GC}^{34}$.

A média de MGT observada em nossas pacientes foi semelhante à média encontrada em um estudo realizado por $\mathrm{Pippa}^{26}$. O IMC foi o único fator determinante independente e significativo para a MGT em nosso estudo.

Na nossa amostra, a dose de GC nos últimos três meses apresentou correlação positiva com a MGT. Curiosamente, a análise multivariada não constatou que esta variável fosse determinante na MGT, a exemplo de outro relato ${ }^{33}$. É possível que o PGT seja um índice mais sensível à utilização de GC. Lembramos que as mulheres do nosso estudo apresentaram sobrepeso. Dessa maneira, é possível que adicionalmente ao TEE, assim como a obesidade, o sobrepeso tenha contribuído de forma positiva no PGT de nossas voluntárias.

\section{ConClusäo}

Este é o primeiro estudo brasileiro que analisa a CC em pacientes com AR e a sua relação com DMO e o uso de GC. Nossos resultados confirmam a diminuição da DMO em pacientes com AR.

A idade e raça branca foram fatores determinantes negativos nas medidas de DMO. O IMC foi um fator positivo em todas as variáveis de $\mathrm{DMO}$ e CC. A duração da doença (AR) também influenciou negativamente a DMOCL nestas pacientes. A utilização de GC modificou a CC, reduzindo de forma rápida a massa muscular e aumentando o percentual de gordura nestas pacientes com a sua utilização prolongada.

A redução da DMO observada em $67,8 \%$ das pacientes sugere a melhoria de medidas para a prevenção e tratamento de osteoporose 
SILVA RG ET AL.

a partir do diagnóstico desta doença. $\mathrm{O}$ achado de valores reduzidos de DMO neste estudo sugere que devam ser aplicadas medidas para a prevenção e tratamento de osteoporose a partir do diagnóstico da AR. Embora não tenhamos evidenciado uma ação deletéria direta do GC na DMO destas mulheres, o seu uso deve ser criterioso em relação ao tempo e à dose.

A ação negativa deste fármaco sobre a MMT nas pacientes é um fator importante que deve ser lembrado. A preservação da massa muscular e o incentivo ao seu aumento devem ser praticados. Este simples procedimento interfere positivamente na DMO e equilíbrio das pacientes, com conseqüente diminuição de quedas e futuras fraturas.

Conflito de interesse: não há.

\section{SUMMARY}

EVALUATION OF BODY COMPOSITION AND BONE MINERAL DENSITY IN WOMEN WITH RHEUMATOID ARTHRITIS

Decrease of bone mass and changes in body composition are common in patients with rheumatoid arthritis (RA) especially in users of glucocorticoids.

OBJECTIVE. To evaluate the bone mineral density (BMD) and its correlation to factors of body composition in women with $R A$.

Methods. BMD and body composition (total and regional) were measured by DXA in 83 patients with rheumatoid arthritis. In addition, a lateral dorsal and lumbar spine $x$-ray was carried out as well as laboratory tests (rheumatoid factor, inflammatory exams). Information about activity of disease, functional class, physical activity and alimentary data were colleted using specific questionnaires.

RESULTS. The prevalence of osteoporosis in menopausal patients was $21.4 \%$ (12 patients), of osteopenia $46.4 \%$ (26 patients) while $32.1 \%$ were normal (18 patients). Osteoporosis was similar in the lumbar spine and the femoral neck. More than half the patients showed low BMD in the lumbar and/or femoral neck. Non Caucasian and premenopausal women had the highest values of BMD averages. The cumulative dose of glucocorticoids for the last two years was a negative determinant of total lean mass. Age had a negative effect on $B M D$ and body composition measurements. BMI showed a positive effect in all $B C$ variables. Functional classes 3 and 4 had a negative effect only on the BMD total. Physical activity had a positive effect on BMD of the total femur. Duration of RA had a negative effect on BMD in the lumbar spine. The GC dose used in the last 3 months showed a negative effect on the total lean mass (MMT) and doses of the last 2 years had a positive effect on the total fat percentage (TFP). Finally, the estrogen exposure time (EET) indicated a positive effect on total fat percentage (TFP).

CONCLUSION. A decreased BMD found in $67.8 \%$ of patients suggests a better approach to prevention and treatment as from diagnosis of the disease. Age and non Caucasian race were negative factors for BMD values, while BMI was a positive factor for all BMD and body composition variables. The disease (RA) also had a negative influence on BMD of these women and use of GC produced changes in body composition, reducing the lean mass and increasing the fat percentage. Despite lack of evidence of a harmful direct action of oral corticoids on BMD, their use should be avoided. Therefore, preserving lean mass and enhancing BMD is important for these patients, in order to decrease fractures and falls. [Rev Assoc Med Bras 2007; 53(2): |35-4I]

KEY WORDS: Body composition. Bone mineral density. Rheumatoid arthritis. Osteoporosis.

\section{RefERÊNCIAS}

I. Blake GM, Wahner HE, Folgeman I. The evaluation of osteoporosis: dual energy $x$-ray and ultrasound in clinical practice. $2^{\text {nd }}$ ed. United Kingdon: The Livery House; 1999. p.313-46.

2. Westhovens J, Nijs J, Taelman V, Dequeker J. Body composition in rheumatoid arthritis. $\mathrm{Br}$ | Rheumatol. 1997;36:444-8.

3. Kremer JM. Epidemiology of rheumatoid arthritis. Rheum Dis Clin North Am. 1995;21:598-604.

4. Roubenoff R, Roubenoff RA, Ward LM, Holland SM, Hellmann DB Rheumatoid cachexia: depletion of lean body mass in rheumatoid arthritis. Possible association with tumor necrosis factor. J Rheumatol. 1992; 19:1 505- 10.

5. Hernadez-Beriain JA, Segura-Garcia C, Rodrigues-Lozano B, Bustabad S, Gantes M, Gozáles T. Undernutrition in rheumatoid arthritis patients with disability. Scand J Rheumatol. 1996;25:383-7.

6. Sambrook PN, Spector TD, Seeman E, Bellamy N, Buchanan RRC, Duffy DL, et al. Osteoporosis in rheumatoid arthritis: a monozygotic co-twin control study. Arthritis Rheum. 1995;38:806-9.

7. Armett F, Edworthy SM, Bloch DA, Mcshane DF, Fries JF, Cooper NS The American Rheumatism Association 1987 revised criteria for the classification of rheumatoid arthritis. Arthritis Rheum. 1988;31:315-24.

8. O'Neill TW, Cooper C, Algra D, Kanis JÁ, Silman AJ, Felsenberg D, et al. Design and development of a questionnaire for use in a multicentre study of vertebral osteoporosis in Europe. The European Vertebral Study. Rheumatol Eur. 1995;24:75-81.

9. Baecke JA, Jurema J, Frijters JE. A short questionnaire for the measurement of habitual activity in epidemiological studies. Am J Clin Nutr. 1982;36:936-42.

I 0. Pereira GAP, Genaro PS, Sarkis KS, Peters BSE, Santos LC, Anitelli T, et al. Validation of a food frequency questionnaire (FFQ) for osteoporotic women. In: The $18^{\text {th }}$ International Congress of Nutrition, Durban (South Africa); 2005. Abstract.

I I. Prevoo MLL, Van't Hof MA, Kupper HH, Van Leeuwen MA, Van de Putte LBA, Van Riel PLCM. Modified disease activity scores that include twenty-eigth-joint counts: development and validation in a prospective longitudinal study of patients with rheumatoid arthritis. Arthritis Rheum. 1995:38:44-8.

1 2. Fries JF, Spitz P, Kraines RG, Holman HR. Mensure of patient outcome in arthritis. Arthritis Rheum. 1980;23:|37-|45.

13. Steinbrocker O, Traeger CH, Batter-Man LC. Therapeutic criteria in rheumatoid atrthritis. JAMA. 1949; 140:659-62.

14. Genant HK, Wu CY, Van Kuijk, Nevitt MC. Vertebral fracture assessment using a semiquantitative technique. J Bone Miner Res. 1993;8:1137-48.

15. National Institutes of Health Consensus Development Panel on osteoporosis prevention, diagnosis and therapy. JAMA. 2001;285:785-95.

16. Riggs BL, Melton LJ. Involutional osteoporosis. N Engl J Med. 1986;314:1676-84.

17. Wasnish RD. Epidemiology of osteoporosis. In: Favus MJ. Primer on the metabolic bone diseases and disorders of mineral metabolism. $3^{\text {rd }}$ ed. Chicago: Lippincott-Raven; 1996. p.249-5I.

18. Bell NH, Shary J, Stevens J, Garza M, Gordon L, Edwards J. Demonstration that bone mass is greater in black than in white children. J Bone Res. 1991;6:719-23. 
19. Aloia JF, Vanish A, Mikhail M, Flaster ER. Body composition by dual energy $X$ - ray absorptiometry in black compared with white women. Osteoporos Int. 1999;10:114-9.

20. World Health Organization. Obesity: presenting and managing the global epidemic report. Geneva; 1998. Avaliable from: http:// www.oms.org.

21. Ribot C, Tremolliers F, Pouilles JM, Bonneu M, Germain F, Louvet JP. Obesity and postmenopausal bone loss: the influence of obesity on vertebral density and bone turnover in postmenopausal women. Bone. 1988;8:327- 31 .

22. Shenstone BD, Marhmoud A, Woodward B. Longitudinal bone mineral density changes in early rheumatoid arthritis. $\mathrm{Br}$ J Rheumatol. 1 994;33:54 I-5.

23. Hansen M, Florescu A, Stoltenberg M, Podenphant J, Pedersen-Zbinden $\mathrm{B}$, Horstey-Petersen $\mathrm{K}$ et al. Bone loss in rheumatoid arthritis. Influence of disease activity, duration of the disease, functional capacity, and corticosteroid treatment. Scand J Rheumatol. 1996;25:367-76.

24. Sinigaglia L, Nevertti A, Mela Q, Bianchi G, Adami S, Frediane B et al. A multicentric cross-sectional study on bone mineral density in rheumatoid arthritis. J Rheumatol. 2000;27:2582-9.

25. Florindo AA, Zerbini CAF, Pippa MG, Latorre MRDO, Jaime PC, Tanaka T. Past and present habitual physical activity and its relationship with bone mineral density in men aged 50 years or more in Brazil. J Gerontol A Biol Sci Med Sci. 2002;57:M654-7.

26. Kimble RB, Srivastava S, Ross FP, Matayosh A, Pacific R. Estrogen deficiency increases the ability of stromal cells to support murine osteoclastogenesis via an interleucin-I and tumor necrosis factor mediated stimulation of macrophage colony-stimulating factor production. J Biol Chem. 1996;271:28890-97.

27. Hall GM, Spector TD, Delmas PD. Markers of bone metabolism in postmenopausal women with rheumatoid arthritis. Effects of corticosteroid and hormone replacement therapy. Arthritis Rheum. 1995;38:902-6.

28. Pippa MG. Influência da composição corpórea na densidade mineral óssea em mulheres brasileiras [dissertação]. São Paulo: Faculdade de Medicina, Universidade São Paulo; 2003.

29. Kroot EJJA, Laan RF, Van Riel PL, Nieuwenhuizen MG, Waal MMC, Pasker de PC. Change in bone mineral density in patients with rheumatoid arthritis during the first decade of disease. Arthritis Rheum. 2001;44:1254-60.
30. Haugeberg G, Uhlig T, Falch JA, Halse JI, Kvien TK. Bone mineral density and frequency of osteoporosis in female patients with rheumatoid arthritis: results from 394 patients in the Oslo Count Rheumatoid Arthritis register. Arthritis Rheum. 2000;43:522-30.

3 I. Toussirot É, Nguyen NU, Dumoulin G, Aubin F, Cédoz JP, Wendling D. Relationship between growth hormone -IGF-I-IGFBP-3 axis and serum leptin levels with bone mass and body composition in patients with rheumatoid arthritis 2005;44:|20-5.

32. Wang K, Hassager C, Ravn P, Wang S, Christiansen C. Total and regional body-composition changes in early postmenopausal women: age -related or menopause related? Am J Clin Nutr. 1994;60:843-8.

33. Natsui K, Tanaka K, Suda M, Yasoda A, Sakuma Y, Ozasa A, et al. High dose glucocorticoid treatment induces rapit loss of trabecular bone mineral density and lean body mass. Osteoporos Int. 2006;17:105-8.

34. Carbonare LD, Arlot ME, Chavassieux PM, Roux JP, Portero NR, Meunier PJ. Comparason of trabecular bone microarchitecture and remodeling in glucocorticoid-induced and postmenopausal osteoporosis. J Bone Miner Res 2001;16:97-103.

35. Adachi JD, Olsynski W, Hanley D. Management of corticosteroidinduced osteoporosis. Semin Arthritis Rheum 2000;22:375-84.

36. Lukert BP, Raisz LG: Glucocorticoid-induced osteoporosis. Rheum Dis Clin North Am 1994;20:629-50.

37. Lane NE. Osteoporosis. Rheum Dis Clin North Am 2001;27:235-53.

38. Lodder MC, de Jong Z, Kostense PJ, Staal K, Hazes JMW, Lems WF, et al. Bone mineral density in patients with rheumatoid arthritis: relation between severity and low bone mineral density. Ann Rheum Dis. 2004;63: I 576-80.

39. Reid IR, Ames R, Evans MC, Sharpe S, Gamble G, France JT, et al. Determinants of total body and regional bone mineral density in normal postmenopausal women - a key role for fat mass. J Clin Endocrinol Metab. |992;75:45-5।.

Artigo recebido: 26/04/06 Aceito para publicação: 06/I I/06 